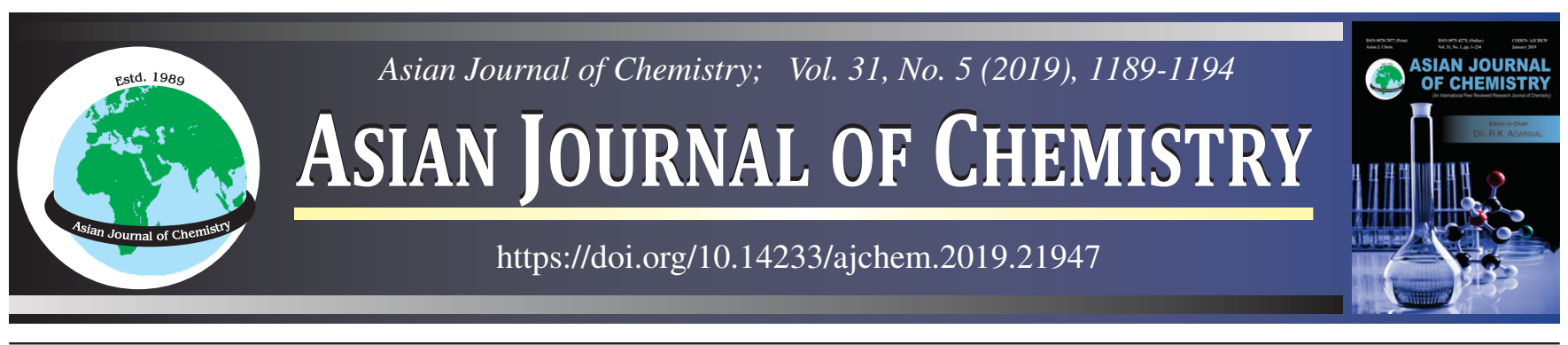

\title{
Water-Mediated Ceric Ammonium Nitrate Catalyzed C-C/C-N Bond Formation: Convenient Access to Polyfunctionalized Pyrazoles via Multicomponent Reaction
}

\author{
Vikas D. Kadu ${ }^{\circledR}$, Mahesh G. Hublikar ${ }^{\circledR}$, Dattatraya G. Raut ${ }^{\circledR}$ and Raghunath B. Bhosale ${ }^{*}$,®
}

Organic Chemistry Research Laboratory, School of Chemical Sciences, Solapur University, Solapur-413255, India

*Corresponding author: Tel/Fax: +91 217 2744770; Email: bhosale62@yahoo.com

Received: 22 January 2019; Accepted: 25 February 2019;

Published online: 28 March 2019;

An efficient multicomponent approach has been developed in the environmentally green aqueous medium for synthesis of substituted polyfunctionalized pyrazoles. The simple and readily available aldehydes, malononitrile and phenylhydrazines substrates afforded polysubstituted imidazoles ( 15 examples) up to $96 \%$ yield. The polyethylene glycol is playing the dual role of solvent and promoter with water in this reaction catalyzed by ceric ammonium nitrate.

Keywords: Multicomponent reaction, Pyrazole, Water-polyethylene glycol media, Ceric ammonium nitrate.

\section{INTRODUCTION}

Pyrazole is a privileged heterocyclic skeleton due to its incorporation into pharmaceuticals, agrochemicals and materials as well as its natural occurrence $[1,2]$. Pyrazoles have been reported to exhibit a wide range of biological properties and immense significance in the pharmacological agents of diverse therapeutic categories [3-5]. Especially, the several blockbuster drugs and pesticides, including celecoxib (celebrex) [6], sildenafil (viagra) [7], zometapine [8], fenpyroximate [9], fipronil [10] and tebufenpyrad [11] have made pyrazole a popular synthetic target. Accordingly, they have been extensively studied in the last few decades as a prominent class with great attention for both pharmaceutical and agricultural benefits.

Fascinatingly, ceric ammonium nitrate (CAN) catalyzed organic transformations are rapidly developing due to its wellknown versatility and stability, commercial availability, reasonably priced and simple handling attracted much attention in carboncarbon and carbon-heteroatom bond formation [12-15]. As might be expected of very powerful one-electron oxidants, the chemistry of cerium(IV) oxidation of organic molecules is dominated by radical and radical cation chemistry [16-19] and emerged as a vital feed-stock in diverse chemical industries [20]. As stated in a recent literature, use of CAN in a site-selective fashion might provide a pathway for building addressable libraries of scaffolds [21-26]. Herein, we are going to report a novel method offering efficient formation of polysubstituted pyrazoles with high productivity from readily available and simple starting materials, namely aldehydes, malononitrile and phenylhydrazines.

\section{EXPERIMENTAL}

All commercially available reagents were used directly without purification unless otherwise stated. All the solvents utilized in the reactions were distilled for purity. ${ }^{1} \mathrm{H}$ NMR and ${ }^{13} \mathrm{C}$ NMR spectra were recorded at 400 and $100 \mathrm{MHz}$, correspondingly. Chemical shifts are expressed in parts per million (ppm) downfield from tetramethylsilane (TMS) as an internal reference standard. Infrared spectra were recorded and band positions are reported. Thin-layer chromatography (TLC) was performed by using commercially prepared $0.25 \mathrm{~mm}$ silica gel plates and visualization was effected with a short wavelength UV lamp (254 nm). The relative proportions of solvents in chromatography solvent mixtures are referred volume to volume ratio.

General procedure for the synthesis of 5-amino-pyrazole4-carbonitriles (4): To a round bottom flask, aldehyde (1) (1 mmol), malononitrile (2) (1.1 mmol), phenylhydrazine (3) (1 mmol), CAN (10 mol \%) and PEG-400: $\mathrm{H}_{2} \mathrm{O}(5 \mathrm{~mL})$ were added with ratio $4: 1(\mathrm{v} / \mathrm{v})$. The resulting reaction mixture was stirred at room temperature for $5 \mathrm{~min}$. After completion of the reaction, stirred reaction mixture further diluted with excess water $(2 \times$

This is an open access journal, and articles are distributed under the terms of the Creative Commons Attribution-NonCommercial-ShareAlike 4.0 (CC BY-NC-SA 4.0) International License which allows readers to freely read, download, copy, distribute, print, search, or link to the full texts of its articles and to use them for any other lawful non-commercial purpose as long as the original source is duly acknowledged. 
$10 \mathrm{~mL}$ ) and then filtered to afford the crude product, which was used further by water washing and drying properly without purification to afford the desired product 4 .

5-Amino-1-phenyl-3-phenyl-1H-pyrazole-4-carbonitrile (4a): Pale yellow solid, yield $93 \%$; m.p.: $159-160{ }^{\circ} \mathrm{C}$; IR (KBr, $\left.v_{\max }, \mathrm{cm}^{-1}\right): 3293,2363,1601,1256 ;{ }^{1} \mathrm{H}$ NMR (400 $\left.\mathrm{MHz}, \mathrm{CDCl}_{3}\right): \delta=7.70$ (m, 2H, ArH), 7.68 (s, 2H, $\left.\mathrm{NH}_{2}\right), 7.40$ (m, 2H, ArH), 7.33 (m, 3H, ArH), 7.29 (m, 2H, ArH), 6.91 (m, $1 \mathrm{H}, \mathrm{ArH}) \mathrm{ppm} ;{ }^{13} \mathrm{C}$ NMR $\left(100 \mathrm{MHz}, \mathrm{CDCl}_{3}\right): \delta 144.68$, 137.33, 135.33, 129.79, 129.33, 129.03, 128.63, 128.44, 128.37, $126.21,120.13,112.79$ ppm; Anal. calcd. (found) $\%$ for $\mathrm{C}_{16} \mathrm{H}_{12} \mathrm{~N}_{4}$ : C, 73.83 (73.84); H, 4.65 (4.63); N, 21.52 (21.54).

5-Amino-1-phenyl-3-p-tolyl-1H-pyrazole-4-carbonitrile (4b): Pink powder solid, yield $90 \%$; m.p.: 117-119 ${ }^{\circ} \mathrm{C}$; IR (KBr, $\left.v_{\max }, \mathrm{cm}^{-1}\right): 3482,3319,3095,2925,2359,1598$, $1415,1257,1127,1113,1096 ;{ }^{1} \mathrm{H}$ NMR (400 MHz, $\left.\mathrm{CDCl}_{3}\right)$ : $\delta 2.41(\mathrm{~s}, 3 \mathrm{H}), 6.91(\mathrm{dd}, J=3.5 \mathrm{~Hz}$ and $J=7.3 \mathrm{~Hz}, 1 \mathrm{H}), 7.15$ (d, $J=7.76 \mathrm{~Hz}, 2 \mathrm{H}), 7.22(\mathrm{~d}, J=7.9 \mathrm{~Hz}, 2 \mathrm{H}), 7.29-7.33(\mathrm{~m}$, $2 \mathrm{H}), 7.59$ (d, $J=7.9 \mathrm{~Hz}, 2 \mathrm{H}), 7.70$ (s, 2H) ppm; ${ }^{13} \mathrm{C}$ NMR $\left(100 \mathrm{MHz}, \mathrm{CDCl}_{3}\right): \delta 153.20,148.82,145.20,138.97,138.14$, $132.95,129.77,129.71,126.62,120.44,113.22,104.65,21.90$ ppm; Anal. calcd. (found) $\%$ for $\mathrm{C}_{17} \mathrm{H}_{14} \mathrm{~N}_{4}$ : C, 74.43 (74.45); H, 5.14 (5.13); N, 20.42 (20.43).

5-Amino-1-phenyl-3-(4-methoxyphenyl)- $1 H$-pyrazole4-carbonitrile (4c): Cream coloured solid, yield $91 \%$; m.p.: 106-108 ${ }^{\circ} \mathrm{C}$; IR (KBr, $\left.v_{\max }, \mathrm{cm}^{-1}\right): 3313,2363,1595,1244 ;{ }^{1} \mathrm{H}$ NMR (400 MHz, DMSO- $\left.d_{6}\right): \delta=7.81\left(\mathrm{~s}, 2 \mathrm{H}, \mathrm{NH}_{2}\right), 7.57$ (d, $J$ $=8.76 \mathrm{~Hz}, 2 \mathrm{H}, \mathrm{ArH}), 7.19(\mathrm{t}, J=7.84,2 \mathrm{H}, \mathrm{ArH}), 7.03(\mathrm{~d}, J=$ $8.00 \mathrm{~Hz}, 2 \mathrm{H}, \mathrm{ArH}), 6.95(\mathrm{~d}, J=8.76 \mathrm{~Hz}, 2 \mathrm{H}, \mathrm{ArH}), 6.71(\mathrm{t}, J$ $=7.22 \mathrm{~Hz}, 1 \mathrm{H}, \mathrm{ArH}), 3.77\left(\mathrm{~s}, 3 \mathrm{H}, \mathrm{OCH}_{3}\right) \mathrm{ppm} ;{ }^{13} \mathrm{C} \mathrm{NMR}(100$ $\left.\mathrm{MHz}, \mathrm{CDCl}_{3}\right): \delta 159.26,145.53,136.53,133.34,129.01$, 128.46, 126.98, 118.28, 114.13, 111.76, 55.13 ppm; Anal. calcd. (found) $\%$ for $\mathrm{C}_{17} \mathrm{H}_{14} \mathrm{~N}_{4} \mathrm{O}: \mathrm{C}, 70.33$ (70.35); $\mathrm{H}, 4.86$ (4.84); N, 19.30 (19.31); O, 5.51 (5.50).

5-Amino-1-phenyl-3-(4-hydroxyphenyl)- $1 \mathrm{H}$-pyrazole4-carbonitrile (4d): Brown solid, yield 89 \%; m.p.: 210-212 ${ }^{\circ} \mathrm{C}$; IR (KBr, $\left.v_{\max }, \mathrm{cm}^{-1}\right): 3398,2181,1688,1257 ;{ }^{1} \mathrm{H}$ NMR $\left(400 \mathrm{MHz}, \mathrm{DMSO}-d_{6}\right): \delta=9.61(\mathrm{~s}, 1 \mathrm{H}, \mathrm{OH}), 7.77(\mathrm{~s}, 2 \mathrm{H}$, $\left.\mathrm{NH}_{2}\right), 7.46(\mathrm{~d}, J=8.56 \mathrm{~Hz}, 2 \mathrm{H}, \mathrm{ArH}), 7.18(\mathrm{t}, J=7.84 \mathrm{~Hz}, 2 \mathrm{H}$, ArH), 7.01 (d, $J=7.72,2 \mathrm{H}, \mathrm{ArH}), 6.78$ (d, $J=8.56,2 \mathrm{H}, \mathrm{ArH})$, 6.69 (t, $J=7.24 \mathrm{~Hz}, 1 \mathrm{H}, \mathrm{ArH}) \mathrm{ppm} ;{ }^{13} \mathrm{C}$ NMR $(100 \mathrm{MHz}$, $\left.\mathrm{CDCl}_{3}\right): \delta 157.64,145.65,137.08,127.12,126.86,118.09$, $115.50,112.04,111.68 \mathrm{ppm}$; Anal. calcd. (found) $\%$ for $\mathrm{C}_{16} \mathrm{H}_{12} \mathrm{~N}_{4} \mathrm{O}: \mathrm{C}, 69.55$ (69.56); H, 4.38 (4.39); N, 20.28 (20.27); $\mathrm{O}, 5.79$ (5.78).

5-Amino-1-(4-chlorophenyl)-3-phenyl-1H-pyrazole-4carbonitrile (4e): Pink solid, yield $92 \%$, m.p.: $133-135{ }^{\circ} \mathrm{C}$; IR $\left(\mathrm{KBr}, v_{\max }, \mathrm{cm}^{-1}\right): 3420,3322,3095,2365,1598,1487$, $1267,1134,1096 ;{ }^{1} \mathrm{H}$ NMR $\left(400 \mathrm{MHz}, \mathrm{CDCl}_{3}\right): \delta=7.70(\mathrm{~s}$, $1 \mathrm{H}), 7.66(\mathrm{~d}, J=7.6 \mathrm{~Hz}, 2 \mathrm{H}), 7.56(\mathrm{~s}, 1 \mathrm{H}), 7.42(\mathrm{t}, J=7.6 \mathrm{~Hz}$, $2 \mathrm{H}), 7.33-7.36(\mathrm{~m}, 1 \mathrm{H}), 7.25(\mathrm{~d}, J=8.4 \mathrm{~Hz}, 2 \mathrm{H}), 7.06(\mathrm{~d}, J=$ $8.8 \mathrm{~Hz}, 2 \mathrm{H}) \mathrm{ppm} ;{ }^{13} \mathrm{C} \mathrm{NMR}\left(100 \mathrm{MHz}, \mathrm{CDCl}_{3}\right): \delta 155.92$, 143.67, 138.37, 135.41, 132.28, 129.52, 129.09, 129.02, 126.60, $125.08,114.22,112.05 \mathrm{ppm}$; Anal. calcd. (found) $\%$ for $\mathrm{C}_{16} \mathrm{H}_{11} \mathrm{ClN}_{4}$ : C, 65.20 (65.22); H, 3.76 (3.75); Cl, 12.03 (12.02); N, 19.01 (19.03).

5-Amino-1-phenyl-3-(4-bromophenyl)-1H-pyrazole-4carbonitrile (4f): Pink solid, yield $93 \%$; m.p.: $165-167{ }^{\circ} \mathrm{C}$;
IR $\left(\mathrm{KBr}, v_{\max }, \mathrm{cm}^{-1}\right): 3302,2371,1592,1254 ;{ }^{1} \mathrm{H}$ NMR $(400$ MHz, DMSO- $\left.d_{6}\right): \delta=7.81\left(\mathrm{~s}, 2 \mathrm{H}, \mathrm{NH}_{2}\right), 7.57(\mathrm{q}, J=7.91 \mathrm{~Hz}$, 4H, ArH), 7.21 (t, $J=7.78 \mathrm{~Hz}, 2 \mathrm{H}, \mathrm{ArH}), 7.06$ (d, $J=7.96$, $2 \mathrm{H}, \mathrm{ArH}), 6.75$ (t, $J=7.27 \mathrm{~Hz}, 1 \mathrm{H}, \mathrm{ArH}) \mathrm{ppm} ;{ }^{13} \mathrm{C}$ NMR (100 $\left.\mathrm{MHz}, \mathrm{CDCl}_{3}\right): \delta 145.12,135.12,134.12,131.51,129.09$, $127.39,120.61,118.95,112.03$ ppm; Anal. calcd. (found) $\%$ for $\mathrm{C}_{16} \mathrm{H}_{11} \mathrm{~N}_{4} \mathrm{Br}$ : C, 56.66 (56.64); H, 3.27 (3.28); Br, 23.56 (23.55); N, 16.52 (16.54).

5-Amino-3-(4-nitrophenyl)-1-phenyl-1H-pyrazole-4carbonitrile (4g): Red solid, yield $95 \%$; m.p.: $164-166{ }^{\circ} \mathrm{C}$; IR $\left(\mathrm{KBr}, \mathrm{v}_{\max }, \mathrm{cm}^{-1}\right): 3465,3355,3105,2354,1610,1417$, 1456, 1345, 1256, 1133, 1108, 1094; ${ }^{1} \mathrm{H}$ NMR (400 MHz, $\left.\mathrm{CDCl}_{3}\right): \delta=8.25(\mathrm{~d}, J=7.6 \mathrm{~Hz}, 2 \mathrm{H}) 8.03(\mathrm{~s}, 1 \mathrm{H}), 7.74-7.77$ (m, 3H), 7.20-7.34 (m, 2H), 7.18 (d, J=7.6 Hz, 2H), $6.96(\mathrm{~s}$, 1H) ppm; ${ }^{13} \mathrm{C}$ NMR $\left(100 \mathrm{MHz}, \mathrm{CDCl}_{3}\right): \delta 156.53,149.56$, $145.26,137.73,135.32,131.72,130.93,129.90,122.78,122.15$, 123.47, 113.43, $112.36 \mathrm{ppm}$; Anal. calcd. (found) \% for $\mathrm{C}_{16} \mathrm{H}_{11} \mathrm{~N}_{5} \mathrm{O}_{2}: \mathrm{C}, 62.95$ (62.96); H, 3.63 (3.61); N, 22.94 (22.96); $\mathrm{O}, 10.48$ (10.47).

5-Amino-3-(2-chlorophenyl)-1-phenyl-1 H-pyrazole-4carbonitrile (4h): Yellow solid, yield $90 \%$; m.p.: 139-141 ${ }^{\circ} \mathrm{C}$; IR (KBr, $\left.v_{\max }, \mathrm{cm}^{-1}\right): 3475,3425,3150,2520,2335,1660$, $1575,765,720 ;{ }^{1} \mathrm{H}$ NMR $\left(400 \mathrm{MHz}, \mathrm{CDCl}_{3}\right): \delta=7.61(\mathrm{~s}, 2 \mathrm{H})$ $7.58(\mathrm{~d}, J=8.4 \mathrm{~Hz}, 2 \mathrm{H}), 7.35(\mathrm{~d}, J=8.4 \mathrm{~Hz}, 2 \mathrm{H}), 7.28-7.32$ $(\mathrm{m}, 2 \mathrm{H}), 7.15(\mathrm{~d}, J=7.6 \mathrm{~Hz}, 2 \mathrm{H}), 6.94(\mathrm{t}, J=7.6 \mathrm{~Hz}, 1 \mathrm{H})$ ppm; ${ }^{13} \mathrm{C}$ NMR (100 MHz, $\left.\mathrm{CDCl}_{3}\right): \delta 154.82,144.87,144.43$, 139.44, 138.52, 137.90, 129.41, 128.54, 127.04, 119.34, 117.25, 112.84, 114.04, $99.48 \mathrm{ppm}$; Anal. calcd. (found) \% for $\mathrm{C}_{16} \mathrm{H}_{11} \mathrm{~N}_{4} \mathrm{Cl}$ : C, 65.20 (65.22); H, 3.76 (3.75); $\mathrm{Cl}, 12.03$ (12.02); N, 19.01 (19.02).

5-Amino-3-(4-cyanophenyl)-1-phenyl- $1 H$-pyrazole-4carbonitrile (4i): Yellow solid, yield $94 \%$; m.p. $158-160{ }^{\circ} \mathrm{C}$; IR (KBr, $\left.v_{\max }, \mathrm{cm}^{-1}\right): 3434,3316,3275,2353,2229,1583$, 1472, 1265, 1155, 1127, 1096; ${ }^{1} \mathrm{H}$ NMR (400 MHz, $\left.\mathrm{CDCl}_{3}\right)$ : $\delta 10.72(\mathrm{~s}, 1 \mathrm{H}, \mathrm{ArH}), 7.85(\mathrm{~s}, 1 \mathrm{H}, \mathrm{NH}), 7.77-7.81(\mathrm{~m}, 4 \mathrm{H}$, ArH), 7.24 (t, $J=7.2 \mathrm{~Hz}, 2 \mathrm{H}, \mathrm{ArH}), 7.13(\mathrm{~d}, J=7.6 \mathrm{~Hz}, 2 \mathrm{H}$, $\mathrm{ArH}), 6.82(\mathrm{t}, J=7.2 \mathrm{~Hz}, 1 \mathrm{H}, \mathrm{ArH}) \mathrm{ppm} ;{ }^{13} \mathrm{C} \mathrm{NMR}(100 \mathrm{MHz}$, $\left.\mathrm{CDCl}_{3}\right): \delta 152.34,146.19,137.83,135.75,129.70,128.15$, 127.09, 126.74, 120.56, 117.92, 115.61, 113.25, $112.82 \mathrm{ppm}$; Anal. calcd. (found) \% for $\mathrm{C}_{17} \mathrm{H}_{11} \mathrm{~N}_{5}$ : C, 71.57 (71.58); H, 3.89 (3.88); N, 24.55 (24.57).

5-Amino-1-(3-chlorophenyl)-3-phenyl-1 $H$-pyrazole-4carbonitrile (4j): Pale yellow solid, yield $92 \%$; m.p.: 172$175{ }^{\circ} \mathrm{C} ;{ }^{1} \mathrm{H}$ NMR $\left(400 \mathrm{MHz}, \mathrm{CDCl}_{3}\right): \delta=7.72-7.68(\mathrm{~m}, 1 \mathrm{H}$, $\mathrm{ArH}), 7.64$ (s, $\left.2 \mathrm{H}, \mathrm{NH}_{2}\right), 7.43-7.35$ (m, 3H, $\left.\mathrm{ArH}\right), 7.22-7.20$ (m, 2H, ArH), 7.18 (s, 1H, ArH), 6.93 (dd, $J=8.4 \& 1.6 \mathrm{~Hz}$, $1 \mathrm{H}, \mathrm{ArH}), 6.86(\mathrm{dd}, J=8.0 \& 1.2 \mathrm{~Hz}, 1 \mathrm{H}, \mathrm{ArH}) \mathrm{ppm} ;{ }^{13} \mathrm{C}$ NMR (100 MHz, $\left.\mathrm{CDCl}_{3}\right): \delta 145.77,138.36,135.20,134.88$, $130.25,128.81,128.67,126.37,119.96,112.80,110.89 \mathrm{ppm}$; Anal. calcd. (found) $\%$ for $\mathrm{C}_{16} \mathrm{H}_{11} \mathrm{~N}_{4} \mathrm{Cl}$ : C, 65.20 (65.22); $\mathrm{H}$, 3.76 (3.78); Cl, 12.03 (12.05); N, 19.01(19.03).

5-amino-3-(5-bromo-2-hydroxyphenyl)-1-(4-bromophenyl)-1H-pyrazole-4-carbonitrile (4k): Yellow solid, yield 87 \%; m.p.: $180-182{ }^{\circ} \mathrm{C} ;{ }^{1} \mathrm{H}$ NMR $\left(400 \mathrm{MHz}, \mathrm{CDCl}_{3}\right): \delta 9.23$ (s, 1H, OH, Ar-OH), 7.83 (s, 1H, ArH), 7.23-7.34 (m, 5H), $6.83(\mathrm{~d}, 3 \mathrm{H}, \mathrm{ArH}) \mathrm{ppm} ;{ }^{13} \mathrm{C} \mathrm{NMR}\left(100 \mathrm{MHz}, \mathrm{CDCl}_{3}\right): \delta 155.9$, 142.71, 139.59, 132.17, 132.10, 131.22, 120.64, 118.31, 113.98, $112.13,111.0$ ppm; Anal. calcd. (found) $\%$ for $\mathrm{C}_{16} \mathrm{H}_{10} \mathrm{~N}_{4} \mathrm{OBr}_{2}$. 
C, 44.27 (44.28); H, 2.32 (2.31); Br, 36.81 (36.82); N, 12.91 (12.92); O, 3.69 (3.70).

5-Amino-3-(5-bromo-2-hydroxyphenyl)-1-(4-chlorophenyl)-1H-pyrazole-4-carbonitrile (4I): Yellow solid, yield $88 \%$; m.p.: $176-178{ }^{\circ} \mathrm{C} ;{ }^{1} \mathrm{H}$ NMR $\left(400 \mathrm{MHz}, \mathrm{CDCl}_{3}\right): \delta 7.79$ (s, 1H, ArH), $7.60(\mathrm{~s}, 1 \mathrm{H}, \mathrm{OH}, \mathrm{Ar}-\mathrm{OH}), 7.27-7.35(\mathrm{~m}, 5 \mathrm{H})$, 6.89-6.93 (m, 3H, ArH) ppm; ${ }^{13} \mathrm{C} \mathrm{NMR}\left(100 \mathrm{MHz}, \mathrm{CDCl}_{3}\right)$ : $\delta$ $156.03,141.61,139.94,132.73,131.47,129.54,125.95$, $120.03,118.53,113.87,111.18 \mathrm{ppm}$; Anal. calcd. (found) \% for $\mathrm{C}_{16} \mathrm{H}_{10} \mathrm{~N}_{4} \mathrm{OBrCl}$ : C, 49.32 (49.32); $\mathrm{H}, 2.59$ (2.61); $\mathrm{Br}, 20.51$ (20.49); Cl, 9.10 (9.12); N, 14.38 (14.40); O, 4.11 (4.13).

5-Amino-1-(3-chlorophenyl)-3-(4-chlorophenyl)-1Hpyrazole-4-carbonitrile (4m): Yellow solid, yield $90 \%$; m.p.: $97-99{ }^{\circ} \mathrm{C} ;{ }^{1} \mathrm{H}$ NMR $\left(400 \mathrm{MHz}, \mathrm{CDCl}_{3}\right): \delta 7.61-7.62(\mathrm{~m}, 2 \mathrm{H}$, $\mathrm{AH}), 7.59$ (s, $\left.2 \mathrm{H}, \mathrm{NH}_{2}\right), 7.35-7.38$ (m, 2H, $\left.\mathrm{ArH}\right), 7.17-7.21$ (m, 2H, ArH), 6.85-6.93 (m, 2H, ArH) ppm; ${ }^{13} \mathrm{C}$ NMR (100 $\left.\mathrm{MHz}, \mathrm{CDCl}_{3}\right): \delta 145.52,136.30,135.23,134.41,133.44$, 130.29, 128.91, 127.45, 120.19, 112.82, 110.92 ppm; Anal. calcd. (found) \% for $\mathrm{C}_{16} \mathrm{H}_{10} \mathrm{~N}_{4} \mathrm{Cl}_{2}: \mathrm{C}, 58.38$ (58.36); $\mathrm{H}, 3.06$ (3.08); Cl, 21.54 (21.56); N, 17.02 (17.04).

5-Amino-3-(2-chloro-4-fluorophenyl)-1-(4-chlorophenyl)-1H-pyrazole-4-carbonitrile (4n): Yellow solid, yield $86 \%$; m.p.: $134-136{ }^{\circ} \mathrm{C} ;{ }^{1} \mathrm{H}$ NMR $\left(400 \mathrm{MHz}, \mathrm{CDCl}_{3}\right): \delta 8.10$ (s, $\left.2 \mathrm{H}, \mathrm{NH}_{2}\right), 7.84(\mathrm{~s}, 1 \mathrm{H}, \mathrm{ArH}), 7.20(\mathrm{~m}, 2 \mathrm{H}, \mathrm{ArH}), 7.1(\mathrm{~s}, 1 \mathrm{H}$, ArH), 7.0 (m, 1H, ArH), 6.90 (m, 2H, ArH) ppm; ${ }^{13} \mathrm{C} \mathrm{NMR}$ $\left(100 \mathrm{MHz}, \mathrm{CDCl}_{3}\right): \delta 163.67,161.16,145.37,135.26,133.65$, $133.29,133.19,130.31,128.74,120.36,116.97,116.73$, $114.93,114.72,112.86,110.96 \mathrm{ppm}$; Anal. calcd. (found) \% for $\mathrm{C}_{16} \mathrm{H}_{9} \mathrm{~N}_{4} \mathrm{Cl}_{2} \mathrm{~F}$ : C, 55.35 (55.36); H, 2.61 (2.60); Cl, 20.42 (20.43); F, 5.47 (5.46); N, 16.14 (16.15).

5-Amino-3-(2-chloro-6-fluorophenyl)-1-(4-chlorophenyl)-1 $H$-pyrazole-4-carbonitrile (40): Yellow solid, yield $85 \%$; m.p.: $75-78{ }^{\circ} \mathrm{C} ;{ }^{1} \mathrm{H}$ NMR (400 MHz, $\left.\mathrm{CDCl}_{3}\right): \delta 7.70$ (m, 2H, ArH), $7.68\left(\mathrm{~s}, 2 \mathrm{H}, \mathrm{NH}_{2}\right), 7.33$ (m, 2H, ArH), 7.29 (m, $1 \mathrm{H}, \mathrm{ArH}), 6.91(\mathrm{~m}, 2 \mathrm{H}, \mathrm{ArH}) \mathrm{ppm} ;{ }^{13} \mathrm{C}$ NMR $(100 \mathrm{MHz}$, $\left.\mathrm{CDCl}_{3}\right): \delta 163.57,161.07,142.87,133.23,133.21,133.18$,
133.08, 129.26, 128.91, 128.87, 125.10, 116.97, 114.89, $114.68,113.93$ ppm; Anal. calcd. (found) $\%$ for $\mathrm{C}_{16} \mathrm{H}_{9} \mathrm{~N}_{4} \mathrm{Cl}_{2} \mathrm{~F}$ : C, 55.35 (55.37); H, 2.61 (2.59); Cl, 20.42 (20.44); F, 5.47 (5.45); N, 16.14 (16.16).

\section{RESULTS AND DISCUSSION}

Our studies directed toward inexpensive, non-toxic cerium(IV) mediated importantly more sustainable reaction media poly(ethylene glycol) (PEG) [27-30] for organic transformations pleasing into account of its popularity. At the outset of our investigation, we selected benzaldehyde (1), malononitrile (2) and phenylhydrazine (3) as model substrates to optimize reaction condition in presence of CAN (20 mol \%) using nonvolatile and nontoxic polyethylene glycol (PEG-200) medium. We observed $58 \%$ of the corresponding desired product 5-amino-1-phenyl-3-phenyl- $1 H$-pyrazole-4-carbonitrile (4a) after $5 \mathrm{~min}$ at room temperature (Table-1, entry 1). To improve the yields of desired product, various types of polyethylene glycol solutions such as PEG-300, PEG-400 and PEG600 were examined and obtained the corresponding product 5-amino-1-phenyl-3-phenyl-1 $\mathrm{H}$-pyrazole-4-carbonitrile (4a) (Table-1, entries 2-4) in yields of 59, 65 and $60 \%$, respectively. Among them, PEG-400 turned out to be the most efficient medium, giving $4 \mathbf{a}$ in higher yield in comparisons to others.

On the other hand, PEG solutions as green reaction media was spurred by several factors $e$.g., green separation chemistry [31-37], study of PEG metal ion coordination [36,38-41], aqueous biphasic solvent (ABS) solvent properties [42-46]. However, the integration of solvent properties of polyethylene glycol (PEG) by aqueous biphasic reactive extraction (ABRE) concept and its additional phase-transfer characteristics into a single efficient system which facilitates the separation of reactants and catalysts from products. Also, environmentally benign properties of these systems in comparison to the current use of organic solvents in extraction and reactive extraction have increased interest. Under these state of affairs, the effect of

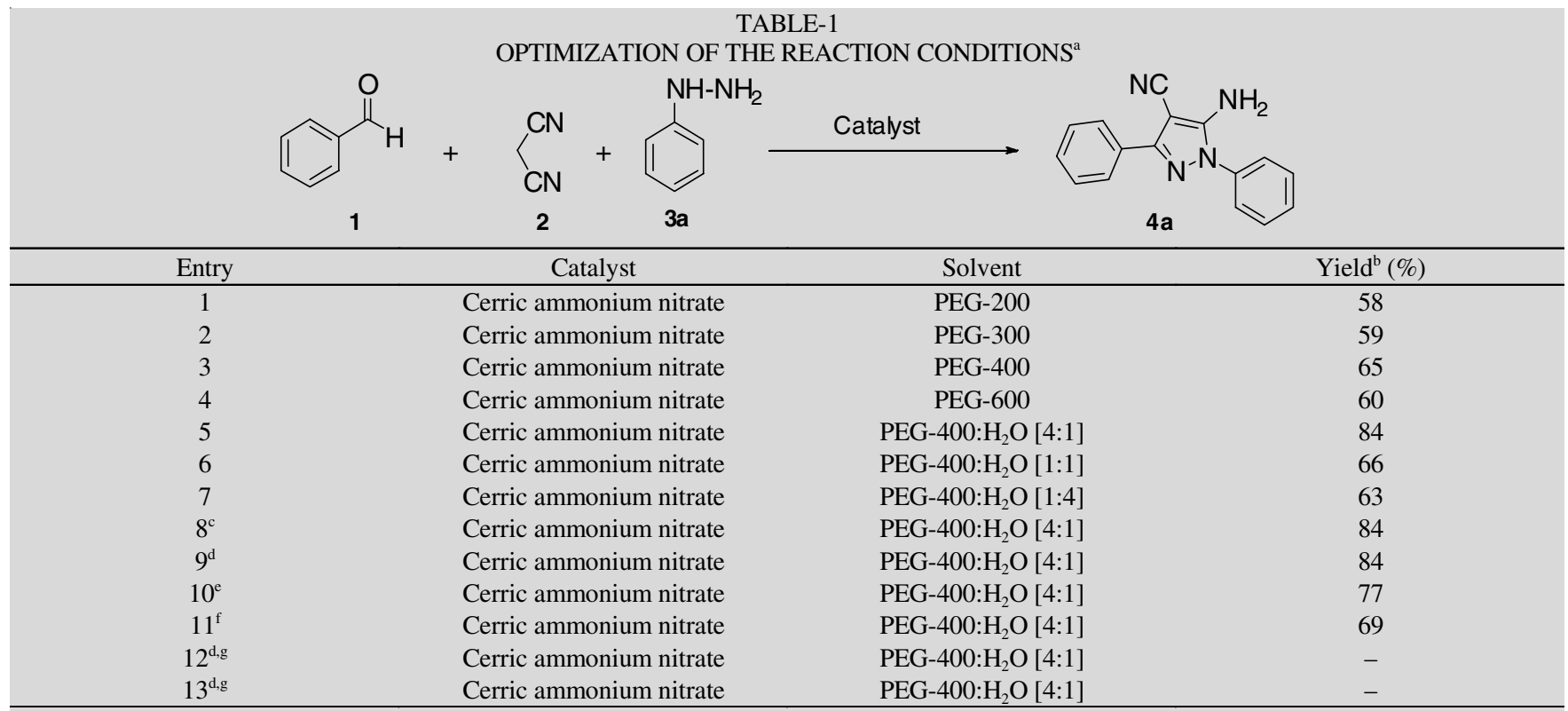

${ }^{a}$ Reaction conditions: 1a $(0.5 \mathrm{mmol}, 1.0$ equiv), 2 ( $0.5 \mathrm{mmol}, 1.0$ equiv), 3a ( $0.5 \mathrm{mmol}, 1.0$ equiv), CAN (20 mol \%) and solvent $(5 \mathrm{~mL})$ stirred for $5 \mathrm{~min}$ at room temperature. ${ }^{\mathrm{b}}$ Isolated yields, ${ }^{\text {cdef }} \mathrm{CAN}(15,10,7$ and $5 \mathrm{~mol} \%),{ }^{\mathrm{g}} \mathrm{Temp} .\left({ }^{\circ} \mathrm{C}\right)=60,80$ in entries 12 and 13 , respectively, CAN $=$ cerric ammonium nitrate, $\mathrm{PEG}=$ polyethylene glycol. 
different PEG-400: $\mathrm{H}_{2} \mathrm{O}(\mathrm{v} / \mathrm{v})$ ratios at room temperature was studied using CAN (20 mol \%). The yields of desired product 4a dramatically increased, i.e. 84, 66 and $63 \%$, respectively (Table-1, entries 5-7). After that, reaction conditions were optimized by variation of catalyst loading and reproduced same results with 15 and $10 \mathrm{~mol} \%$ of CAN as well (Table-1, entries $8-9)$. In next, we find that further decrease in catalyst loading from 10 to $5 \mathrm{~mol} \%$ desired product was obtained in lower yields (Table-1, entries 10-11). Furthermore, the same results were obtained for different temperatures such as $60,80{ }^{\circ} \mathrm{C}$ (Table-1, entries 12-13), respectively.

With the optimized reaction condition in hand, we investigated the various functional group substituted on the benzene ring of aromatic benzaldehydes (Fig. 1), we found that compounds $\mathbf{4 b - 4 d}$ have electron-donating functional groups bearing benzaldehydes 1b-1d (4-Me, 4-MeO and 4-OH) afforded slightly higher yields. Then, considerably decreased in the yields (com- pounds $\mathbf{4 e - 4 h}$ ) for electron withdrawing functional group was observed. The scope of the reaction was further extended to different aromatic aldehydes with malononitrile and substituted phenylhydrazines under the optimized reaction conditions and the products obtained (compounds 4i-4o). The electronic effect introduced by the substitution of the aromatic aldehydes had insignificant influence on the yields of the corresponding pyrazole derivatives. The products were obtained as white to dark yellow solid and data matches well with the literature values. In general, the CAN catalyzed progression is taking place well under mild conditions for a wide range of aldehydes and phenylhydrazines moieties with electron-withdrawing and electrondonating substituents for synthesis of polysubstituted pyrazoles in good to excellent yields.

On the basis of the above-mentioned experimental results, a possible reaction mechanism is proposed for the developed multicomponent protocol in presence of CAN as an activator

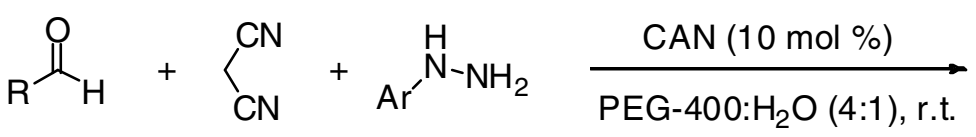

1<smiles>N#Cc1c(-c2ccccc2)nn(-c2ccccc2)c1N</smiles>

4a<smiles>N#Cc1c(-c2ccc(Cl)cc2)nn(-c2ccccc2)c1N</smiles>

$4 e$<smiles>Cc1ccc(-c2nn(-c3ccccc3)c(N)c2C#N)cc1</smiles>

4b<smiles>N#Cc1c(-c2ccc(Br)cc2)nn(-c2ccccc2)c1N</smiles>

$4 f$<smiles>N#Cc1c(-c2ccccc2)nn(-c2cccc(Cl)c2)c1N</smiles>

4j<smiles>COc1ccc(-c2nn(-c3ccccc3)c(N)c2C#N)cc1</smiles>

4c<smiles>N#Cc1c(-c2ccc([N+](=O)[O-])cc2)nn(-c2ccccc2)c1N</smiles><smiles>N#Cc1c(-c2cc(Br)ccc2O)nn(-c2ccc(Br)cc2)c1N</smiles><smiles>N#Cc1ccc(-c2nn(-c3ccccc3)c(N)c2C#N)cc1</smiles>

$4 \mathbf{i}$<smiles>[R]c1nn([Al])c(N)c1C#N</smiles>

4<smiles>N#Cc1c(-c2ccc(O)cc2)nn(-c2ccccc2)c1N</smiles>

4d<smiles>N#Cc1c(-c2ccccc2Cl)nn(-c2ccccc2)c1N</smiles><smiles>N#Cc1c(-c2ccc(F)cc2Cl)nn(-c2cccc(Cl)c2)c1N</smiles><smiles>N#Cc1c(-c2c(F)cccc2Cl)nn(-c2ccc(Cl)cc2)c1N</smiles>

Reaction conditions: 1 (1.1 mmol), 2 (1.0 mmol), (1.0 mmol), 3 (1.0 mmol), $10 \mathrm{~mol} \% \mathrm{CAN}$ in $5 \mathrm{~mL}$ PEG-400: $\mathrm{H}_{2} \mathrm{O}(4: 1)$ at room temperature

Fig. 1. Synthesis of substituted polyfunctionalized pyrazoles 
and oxidizing agent depicted in Scheme-I (4a as an example). Initially, aromatic benzaldehyde (1a) reacted with activated nitrile group from malononitrile (2) in presence of water and polyethylene glycol 400 (PEG-400: $\mathrm{H}_{2} \mathrm{O}$ ) medium with ratio $4: 1(\mathrm{v} / \mathrm{v})$, leads to the formation of the benzylidene-malononitrile adduct (5) via condensation with the formation of $\mathrm{C}=\mathrm{C}$ bond. Afterward, phenylhydrazine (3), to be able to react easily with formed adduct benzylidene-malononitrile (5) gives rise to intermediate (6a). On the other hand, aromatic benzaldehyde (1a) and phenylhydrazine (3) possibly leads to the formation of intermediate imine (7), subsequently reacts with malononitrile (2) furnishes 2-(phenyl(2-phenylhydrazinyl)methyl)malononitrile (6b) via Michael addition. Both of the forms, $\mathbf{6 a}$ and $\mathbf{6 b}$ have equilibrium via a tautomeric 1,3-H shift. Such equilibrium responsible to interconvert them into one another ascertained that reaction time and yield of the product are not seemed to be dependent on the order of addition of reactants in reaction. As a result, both $(\mathbf{6 a})$ and $(\mathbf{6 b})$ affords five-membered ring construction of 5-imino-1,3-diphenylpyrazolidine-4-carbonitrile (9) via $\mathrm{C}-\mathrm{N}$ bond formation. In next step, owing to the imine-enamine tautomerism, 5-imino-1,3diphenylpyrazolidine-4-carbonitrile (9) get transform to 2,3dihydropyrazole derivative (10) with tautomeric proton shift. Simultaneously, 2,3-dihydropyrazole derivative (10) converts to the product (4a) efficiently with $\mathrm{C}-\mathrm{C}$ and $\mathrm{C}-\mathrm{N}$ bond formation in presence of CAN under aerobic oxidation (Scheme-I).

\section{Conclusion}

In summary, an efficient and regioselective method has been developed having facile and practical reaction condition to furnish polysubstituted pyrazoles, providing easy access to the broad library. In this, the intramolecular $\mathrm{C}-\mathrm{N}$ bond formation via oxidation of $\mathrm{C}-\mathrm{H}$ and $\mathrm{N}-\mathrm{H}$ bonds in construction of nitrogen-containing heterocyclic ring system has various advantages, such as direct functionalization of $\mathrm{C}-\mathrm{H}$ bonds with NHR groups without pre-activation of the reaction centers;<smiles>CCOC(C#N)C=CN</smiles>

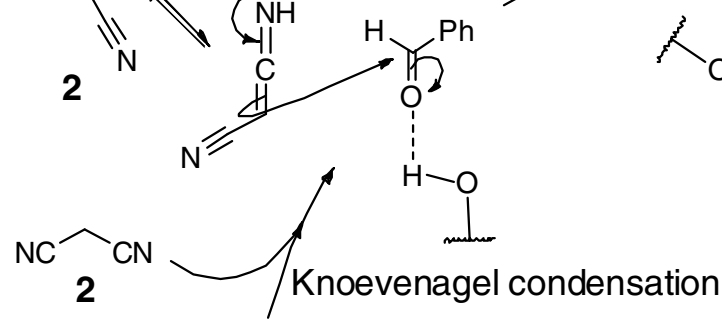<smiles>O=Cc1ccccc1</smiles>
1 a<smiles>CC(CO)CCCNNc1ccccc1</smiles>

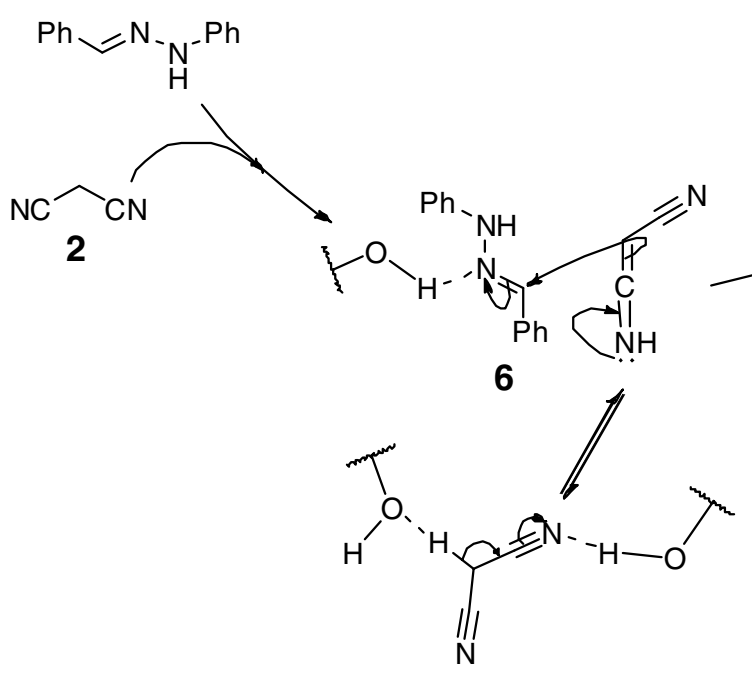<smiles>CCCC(=C(C#N)C1CCN1COCC)c1ccccc1</smiles>

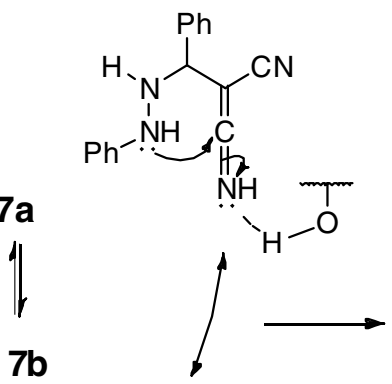

$7 \mathbf{b}$

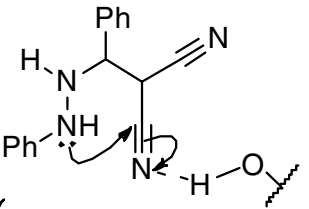

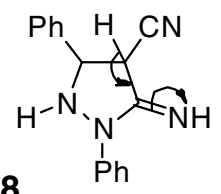

8<smiles>[3H]I</smiles>
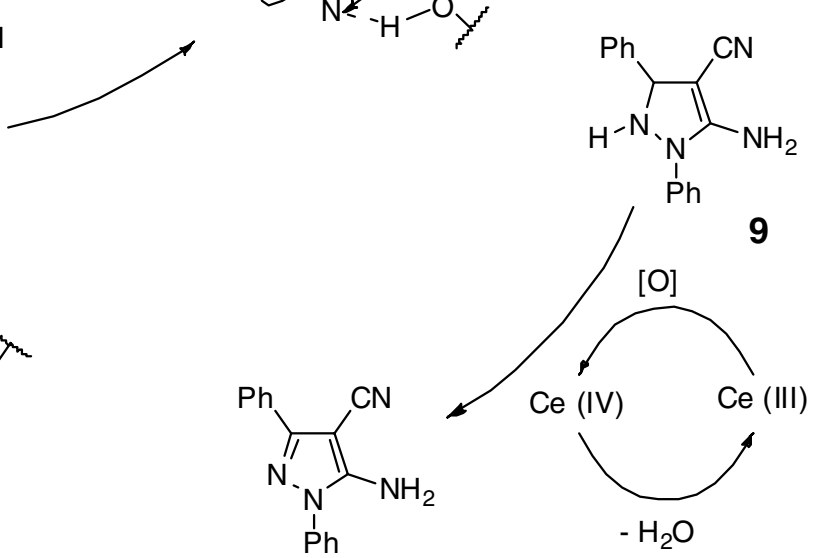

$4 a$

Scheme-I: Plausible reaction mechanism 
facilitative preparation of structurally diverse substrates and no generation of the unwanted stoichiometric amounts of byproducts derived from the leaving groups in substrates for the classic coupling reaction. Hence, it is general, inexpensive and environmentally benign approach having operational simplicity (reaction, work-up and purification) could make it potentially attractive for library construction of polyfunctionalized pyrazoles, particularly in the area of pharmaceuticals.

\section{CONFLICT OF INTEREST}

The authors declare that there is no conflict of interests regarding the publication of this article.

\section{REFERENCES}

1. J.-J. Liu, M. Zhao, X. Zhang, X. Zhao and H.-L. Zhu, Mini Rev. Med. Chem., 13, 1957 (2013); https://doi.org/10.2174/13895575113139990078.

2. V. Kumar, K. Kaur, G.K. Gupta and A.K. Sharma, Eur. J. Med. Chem., 69, 735 (2013); https://doi.org/10.1016/j.ejmech.2013.08.053.

3. J. Dwivedi, S. Sharma, S. Jain and A. Singh, Mini Rev. Med. Chem., 18, 918 (2018); https://doi.org/10.2174/1389557517666170927160919.

4. S. Ganguly and S.K. Jacob, Mini Rev. Med. Chem., 17, 959 (2017); https://doi.org/10.2174/1389557516666151120115302.

5. M.F. Khan, M.M. Alam, G. Verma, W. Akhtar, M. Akhter and M. Shaquiquzzaman, Eur. J. Med. Chem., 120, 170 (2016); https://doi.org/10.1016/j.ejmech.2016.04.077.

6. T.D. Penning, J.J. Talley, S.R. Bertenshaw, J.S. Carter, P.W. Collins, S. Docter, M.J. Graneto, L.F. Lee, J.W. Malecha, J.M. Miyashiro, R.S. Rogers, D.J. Rogier, S.S. Yu, G.D. Anderson, E.G. Burton, J.N. Cogburn, S.A. Gregory, C.M. Koboldt, W.E. Perkins, K. Seibert, A.W. Veenhuizen, Y.Y. Zhang and P.C. Isakson, J. Med. Chem., 40, 1347 (1997); https://doi.org/10.1021/jm960803q

7. N.K. Terrett, A.S. Bell, D. Brown and P. Ellis, Bioorg. Med. Chem. Lett., 6, 1819 (1996); https://doi.org/10.1016/0960-894X(96)00323-X.

8. H.A. DeWald, S. Lobbestael and B.P.H. Poschel, J. Med. Chem., 24, 982 (1981);

https://doi.org/10.1021/jm00140a013.

9. M. Kim, C. Sim, D. Shin, E. Suh and K. Cho, Crop Prot., 25, 542 (2006); https://doi.org/10.1016/j.cropro.2005.08.010.

10. R.E. Sammelson and J.E. Casida, J. Org. Chem., 68, 8075 (2003); https://doi.org/10.1021/jo034520z.

11. D. Marcic, Exp. Appl. Acarol., 36, 177 (2005); https://doi.org/10.1007/s10493-005-3579-2.

12. V. Nair, J. Mathew, J. Prabhakaran and R. Ruzziconi, Chem. Soc. Rev., 26, 127 (1997); https://doi.org/10.1039/CS9972600127.

13. V. Nair, L. Balagopal, R. Rajan and J. Mathew, Acc. Chem. Res., 37, 21 (2004); https://doi.org/10.1021/ar030002z.

14. S.B. Panicker, L.G. Nair, T.G. George and A. Augustine, Synlett., 2003, 156 (2003); https://doi.org/10.1055/s-2003-36775.

15. A. Dhakshinamoorthy, Synlett, 2005, 3014 (2005); https://doi.org/10.1055/s-2005-921893.

16. G.A. Molander, Chem. Rev., 92, 29 (1992); https://doi.org/10.1021/cr00009a002.

17. B.B. Nayak, S. Sahu, S. Patel, S. Dash and B.K. Mishra, Indian J. Chem., 47A, 1486 (2008).

18. V. Nair, L. Balagopal, R. Rajan and J. Mathew, Acc. Chem. Res., 37, 21 (2004); https://doi.org/10.1021/ar030002z.

19. V. Nair and A. Deepthi, Chem. Rev., 107, 1862 (2007); https://doi.org/10.1021/cr068408n.

20. T.J. Demars, M.K. Bera, S. Seifert, M.R. Antonio and R.J. Ellis, Angew. Chem. Int. Ed., 54, 7534 (2015); https://doi.org/10.1002/anie.201502336.
21. X. Zeng, S. Ji and S. Wang, Tetrahedron, 61, 10235 (2005); https://doi.org/10.1016/j.tet.2005.08.040.

22. S.Y. Wang and S.J. Ji, Tetrahedron, 62, 1527 (2006); https://doi.org/10.1016/j.tet.2005.11.011.

23. G. Savitha and P.T. Perumal, Tetrahedron Lett., 47, 3589 (2006); https://doi.org/10.1016/j.tetlet.2006.03.046.

24. V. Nair, K. Mohanan, T.D. Suja and E. Suresh, Tetrahedron Lett., 47, 705 (2006); https://doi.org/10.1016/j.tetlet.2005.11.115.

25. R. Varala, R. Enugala, S. Nuvula and S.R. Adapa, Synlett, 2006, 1009 (2006); https://doi.org/10.1055/s-2006-939066.

26. V. Sridharan, C. Avendaño and J.C. Menéndez, Tetrahedron, 63, 673 (2007); https://doi.org/10.1016/j.tet.2006.11.002.

27. D.E. Bergbreiter, J. Tian and C. Hongfa, Chem. Rev., 109, 530 (2009); https://doi.org/10.1021/cr8004235.

28. N.R. Candeias, L.C. Branco, P.M.P. Gois, C.A.M. Afonso and A.F. Trindade, Chem. Rev., 109, 2703 (2009);

https://doi.org/10.1021/cr800462w.

29. Z. Andrade and L. Alves, Curr. Org. Chem., 9, 195 (2005); https://doi.org/10.2174/1385272053369178.

30. J. Chen, S.K. Spear, J.G. Huddleston and R.D. Rogers, Green Chem., 7, 64 (2005);

https://doi.org/10.1039/b413546f.

31. R.D. Rogers, A.H. Bond, C.B. Bauer, J. Zhang, M.L. Jezl, D.M. Roden, S.D. Rein and R.R. Chomko, eds.: R.D. Rogers and M.A. Eiteman, Aqueous Biphasic Separations: Biomolecules to Metal Ions, Plenum: New York (1995).

32. J.G. Huddleston, H.D. Willauer, K.R. Boaz and R.D. Rogers, J. Chromatogr. B: Biomed. Appl., 711, 237 (1998); https://doi.org/10.1016/S0378-4347(97)00662-2.

33. R.D. Rogers, H.D. Willauer, S.T. Griffin and J.G. Huddleston, J. Chromatogr. B: Biomed. Appl., 711, 255 (1998); https://doi.org/10.1016/S0378-4347(97)00661-0.

34. R.D. Rogers, A.H. Bond, J. Zhang and C.B. Bauer, Appl. Radiat. Isot., 47, 497 (1996); https://doi.org/10.1016/0969-8043(95)00335-5.

35. R.D. Rogers, A.H. Bond, C.B. Bauer, J. Zhang, S.D. Rein, R.R. Chomko and D.M. Roden, Solvent Extr. Ion Exch., 13, 689 (1995); https://doi.org/10.1080/07366299508918298.

36. R.D. Rogers, A.H. Bond and C.B. Bauer, Sep. Sci. Technol, 28, 139 (1993); https://doi.org/10.1080/01496399308019483.

37. R.D. Rogers, C.B. Bauer and A.H. Bond, Sep. Sci. Technol., 30, 1203 (1995); https://doi.org/10.1080/01496399508010341.

38. R.D. Rogers, A.H. Bond and J.L. Wolff, J. Coord. Chem., 29, 187 (1993); https://doi.org/10.1080/00958979308037425.

39. R.D. Rogers, A.H. Bond, S. Aguinaga and A. Reyes, J. Am. Chem. Soc., 114, 2967 (1992); https://doi.org/10.1021/ja00034a032.

40. R.D. Rogers, A.H. Bond and D.M. Roden, Inorg. Chem., 35, 6964 (1996); https://doi.org/10.1021/ic960587b.

41. R.D. Rogers, J. Zhang and C.B. Bauer, J. Alloys Compd., 249, 41 (1997); https://doi.org/10.1016/S0925-8388(96)02640-0.

42. H.D. Willauer, J.G. Huddleston and R.D. Rogers, Ind. Eng. Chem. Res., 41, 1892 (2002); https://doi.org/10.1021/ie010598z.

43. J.G. Huddleston, H.D. Willauer and R.D. Rogers, J. Chromatogr. B: Biomed. Sci. Appl., 743, 137 (2000); https://doi.org/10.1016/S0378-4347(00)00230-9.

44. H.D. Willauer, J.G. Huddleston, S.T. Griffin and R.D. Rogers, Sep. Sci. Technol., 34, 1069 (1999); https://doi.org/10.1080/01496399908951081.

45. H.D. Willauer, J.G. Huddleston and R.D. Rogers, Ind. Eng. Chem. Res., 41, 2591 (2002); https://doi.org/10.1021/ie0107800.

46. J.G. Huddleston, H.D. Willauer, R.D. Rogers, R.D. Rogers, P. Gaillard, P.-A. Carrupt, B. Testa and M.H. Abraham, Phys. Chem. Chem. Phys., 4, 4065 (2002); https://doi.org/10.1039/B203018G. 